\title{
Cross-platform Holder Kit for a Real 3D Correlative Tomography and Microscopy
}

Bartlomiej Winiarski ${ }^{1,2}$, Grzegorz Pyka ${ }^{1,3}$, Ali Chirazi ${ }^{4}$ and Daniel Lichau ${ }^{4}$

${ }^{1}$ Thermo Fisher Scientific, Brno, Jihomoravsky kraj, Czech Republic, ${ }^{2}$ The Henry Royce Institute, School of Materials, The University of Manchester, Manchester, England, United Kingdom, ${ }^{3}$ Université Catholique de Louvain, Louvain, Brussels Hoofdstedelijk Gewest, Belgium, ${ }^{4}$ Thermo Fisher Scientific, Bordeaux, Languedoc-Roussillon, France

*Corresponding author: Bartlomiej.Winiarski@thermofisher.com

Three-dimensional (3D) correlative multi-scale tomography and microscopy (CMT) for the same regions of interest and at different length scales allows spatial and temporal registration in two and three dimensions of many imaging modalities. For example, visible light imaging, electron and cathodoluminescence imaging, ion microscopy, EBSD and EDS analytics, x-ray tomography, magnetic resonance imaging, Raman, atomic force microscopy, SIMS, etc. information may be merged bringing a new insight on the same region of interest.

CMT in materials [1-5] and life sciences [6] uses number of micro/nano x-ray computed tomography $(\mu \mathrm{CT})$ scanners, optical microscopes, LMIS and Plasma DualBeam and Laser Plasma DualBeam microscopes, transmission electron microscopes (TEM), nano computed tomography (CT) in TEM and SEM, etc. All these apparatuses are self-contained and have different requirement for sample preparation, size, fixture, handling and transferring etc., thus direct sample transfer between is very limited or entirely prohibited. The field of correlative microscopy is broad and relatively young, thus the research equipment used up to date is not designed within a framework of the CMT. Each research center has own custom solution that is very limited but suits the defined correlative workflow. While commercially available correlative microcopy transfer means are designed for 2D data acquisition, e.g. optical microscopy (OM) to SEM, SEM to Raman, etc. with extension to, somewhat 3D, superficial surface layers (dozens of microns) when using LMIS FIB-SEM. The advent of Plasma FIBs allowed accessing region of interest berried few hundreds of microns below the surface [1], while commercially available femtosecond Laser Plasma FIB $[4,7]$ with ease can access locations well below $1 \mathrm{~mm}$ in a dozen of minutes. The first real $3 \mathrm{D}$ correlative tomography and microscopy purpose designed cross-platform ( $\mu \mathrm{CT} \Leftrightarrow$ DualBeams) sample transfer and Maps-based correlation solution was recently proposed by Winiarski at al. [4] and is already adopted in academic and industrial environments.

In this contribution we present new HeliScan $\mu \mathrm{CT} \Leftrightarrow$ DualBeams cross-platform holder kit. This setup aims achieving highest $\mu \mathrm{CT}$ resolution $(<400 \mathrm{~nm})$, allowing real 3D correlative tomography and microscopy by accessing region of interest in any location of the specimen and collecting quantitative information using 3D-EBSD/EDS and residual stresses by 3D FIB-SEM-DIC micro-hole drilling method. Our correlative hardware solution is supported by Maps-based [4] and Avizo-based workflows [1] and is compatible with the inert gas transfer hardware and the cryo stage. 


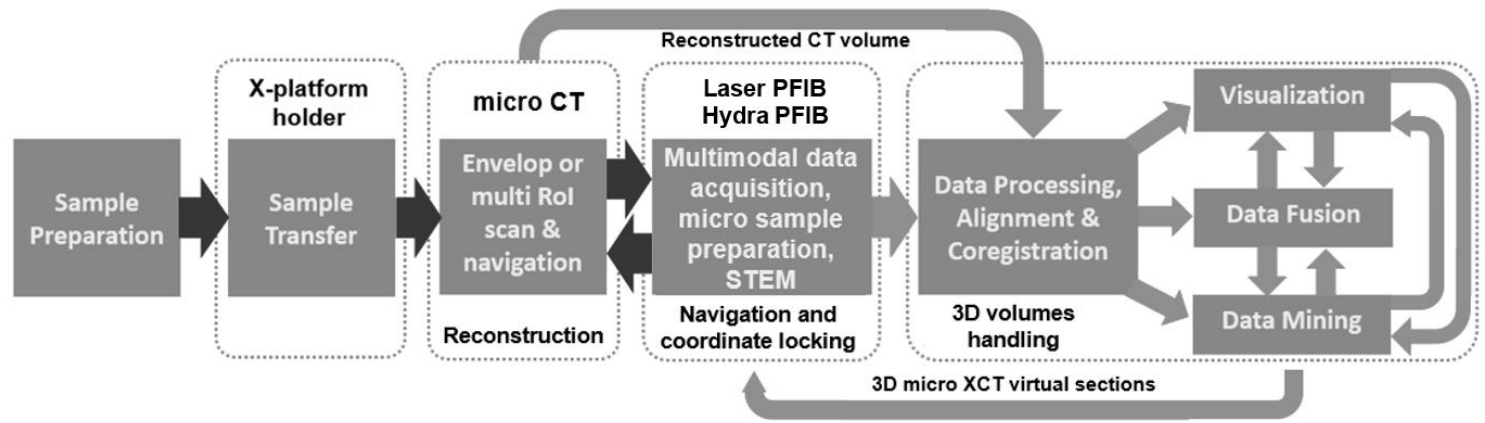

Figure 1. Diagram shows the correlative microscopy/tomography (CMT) Maps-based workflow for material science. Dark arrows indicate sample transfer, while gray arrows show data transfer. CMT is based on dedicated suite of instruments and software.
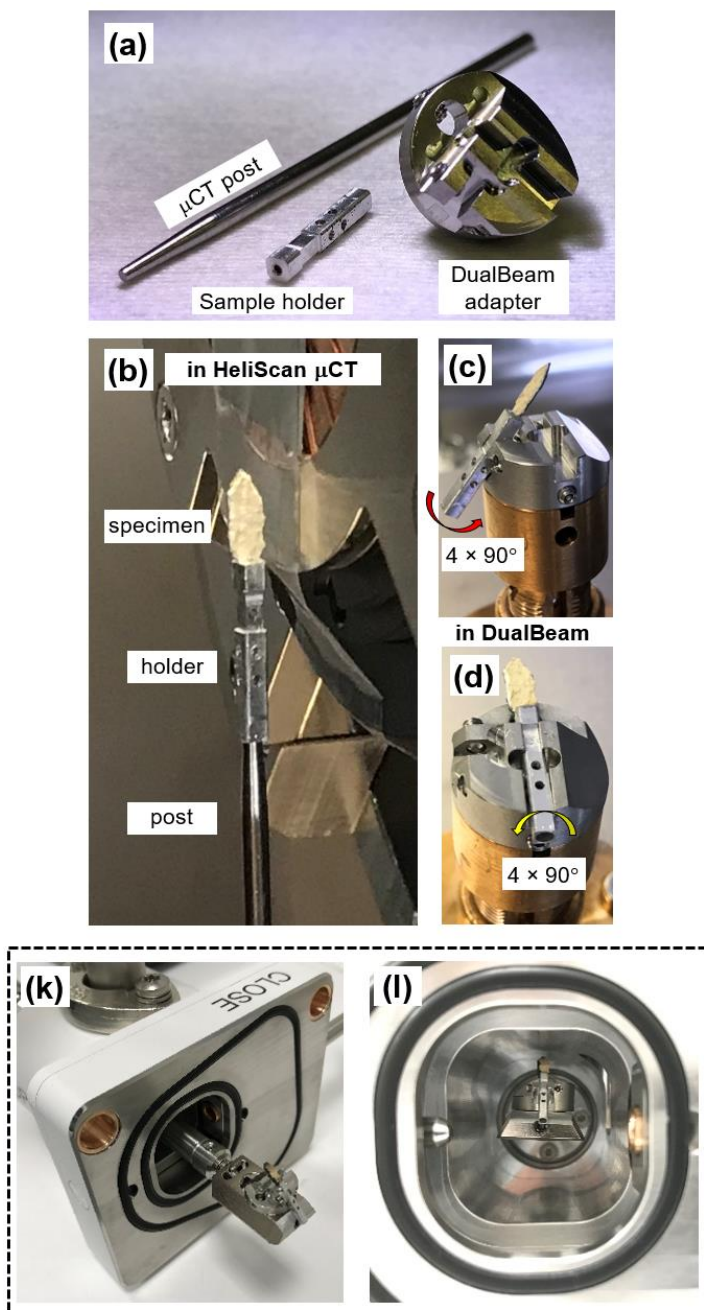
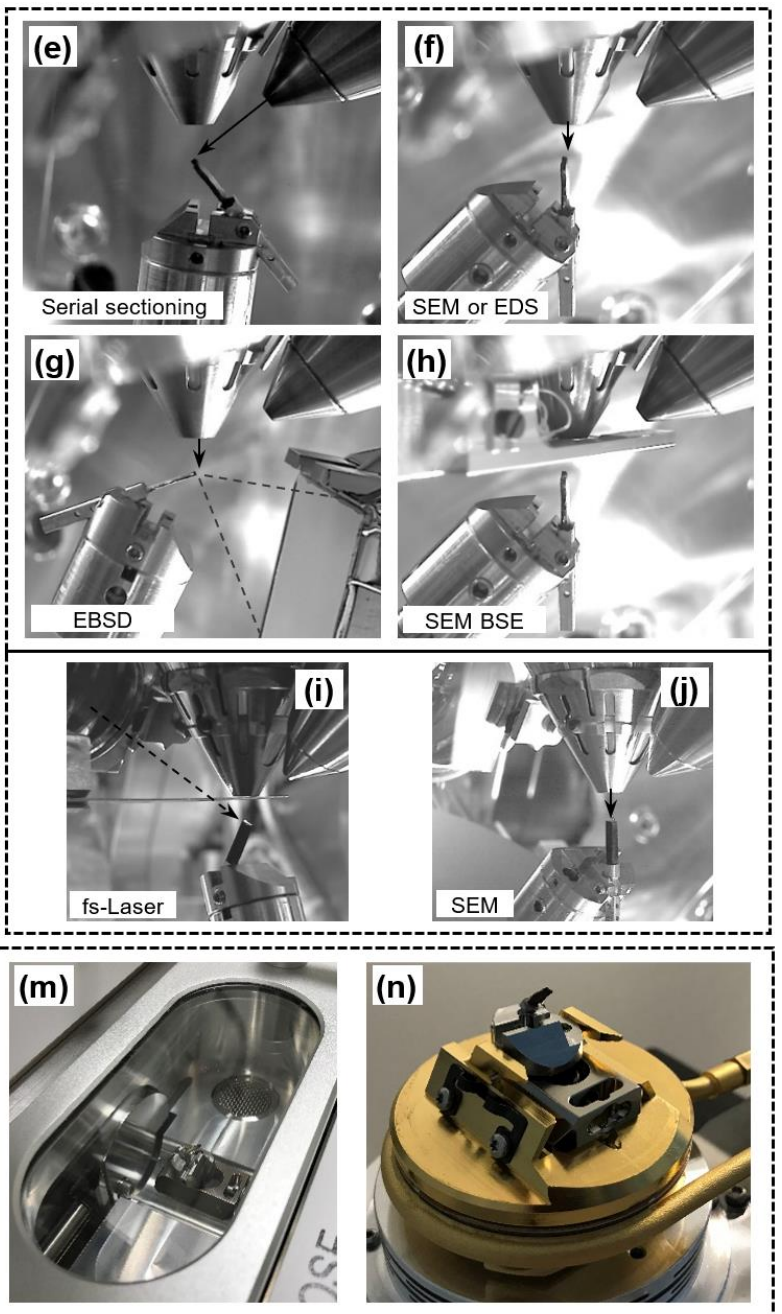

Figure 2. Shows the cross-platform holder kit and its application in various platforms. (a) the kit; (b) in HeliScan $\mu \mathrm{CT}$; (c) and (d) in DualBeam configuration at $45^{\circ}$ and $0^{\circ}$ holder position; (e) - (h) serial sectioning with 3D EBSD and 3D EDS; (i) - (j) serial sectioning in Laser PFIB; (k) - (m) the inert gas transfer hardware of a DualBeam; (n) on the cryo stage.

References 
[1] B Winiarski at al. Supplement of Microscopy and Microanalysis 152 (2017), p. S4-S9.

[2] B Winiarski at al. Microscopy and Microanalysis 23 (S1/2017), p. 342-343.

[3] TL Burnett et al. Scientific Reports 4 (2014), p. 4711.

[4] B Winiarski at al. Microscopy and Microanalysis 25(S2)-2019:870-871.

[5] R Moroni, et al. Scientific Reports 6 (2016), p. 30109.

[6] S Meschini. European journal of histochemistry 2017 EJH 61(s4):1

[7] SJ Randolph et al. Journal of Vacuum Science \& Technology B36 (2018), p. 06 JB01. 